\title{
ROTATION INVARIANT TEXTURE CLASSIFICATION USING ADAPTIVE LBP WITH DIRECTIONAL STATISTICAL FEATURES
}

\author{
Zhenhua Guo ${ }^{1}$, Lei Zhang ${ }^{2}$, David Zhang ${ }^{2}$ and Su Zhang ${ }^{3}$ \\ ${ }^{1}$ Graduate School at Shenzhen, Tsinghua University \\ ${ }^{2}$ Department of Computing, The Hong Kong Polytechnic University \\ ${ }^{3}$ Department of Biomedical Engineering, Shanghai Jiaotong University
}

\section{ABSTRACT}

Local Binary Pattern (LBP) has been widely used in texture classification because of its simplicity and computational efficiency. Traditional LBP codes the sign of the local difference and uses the histogram of the binary code to model the given image. However, the directional statistical information is ignored in LBP. In this paper, some directional statistical features, specifically the mean and standard deviation of the local absolute difference are extracted and used to improve the LBP classification efficiency. In addition, the least square estimation is used to adaptively minimize the local difference for more stable directional statistical features, and we call this scheme the adaptive LBP (ALBP). By coupling the directional statistical features with ALBP, a new rotation invariant texture classification method is presented. Experiments on a large texture database show that the proposed texture feature extraction and classification scheme could significantly improve the classification accuracy of LBP.

Index Terms - LBP, Rotation Invariance, LSE

\section{INTRODUCTION}

Texture analysis is an active research topic in the fields of computer vision and pattern recognition. Generally speaking, it involves four basic problems: classifying images based on texture content; segmenting an image into regions of homogeneous texture; synthesizing textures for graphics applications; and establishing shape information from texture cue [1].

In the early stage, researchers devoted themselves into extracting statistical feature to classify texture images, such as the co-occurrence matrix method [2] and the filtering based methods [3]. These methods could achieve good classification results if the training and testing samples have similar or identical orientation. To meet some real application requirements, rotation invariance is a critical issue to be solved. Kashyap and Khotanzad [4] were among the first researchers to study rotation-invariant texture classification by using a circular autoregressive model. Later, many other models were explored, including the multiresolution autoregressive model [5], and hidden Markov model [6]. Jafari-Khouzani and Soltanian-Zadeh proposed to use Radon transform to estimate the texture orientation and extract wavelet energy features for texture classification [8]. Recently, Varma and Zisserman [9] proposed a statistical learning based algorithm, where a rotation invariant texton library is first built from a training set and then an unknown texture image is classified according to its texton distribution. Scale and affine invariant texture classification is an active research topic, and some pioneer work have been done by using fractal analysis [11] and affine adaptation [10].

In [7], Ojala et al. proposed to use the Local Binary Pattern (LBP) histogram for rotation invariant texture classification. LBP is a simple but efficient operator to describe local image patterns. The LBP based methods have achieved good classification results on representative texture databases. Since LBP could not fully represent the image local spatial structure, the contrast (the variance of local image) was proposed to combine with LBP for a joint distribution [7]. However, the contrast has two main limitations. First, contrast is an isotropic measurement, while texture images may contain clear orientation. Thus the contrast fails to represent such information. Second, contrast needs a quantization procedure to combine with LBP. This quantization step may fail to well represent the feature space when the number of training samples is limited.

In this paper, we propose to incorporate the directional statistical information for rotation invariant texture classification. The distribution of local difference between each pixel and its neighborhoods in each direction is modeled by the mean and standard deviation. To reduce the estimation error of local difference, an adaptive LBP (ALBP) is developed using least square estimation (LSE). Moreover, the coefficients of the LSE in the ALBP could provide additional information for classification.

The rest of the paper is organized as follows. Section 2 briefly reviews the LBP. Section 3 presents the ALBP and the directional statistical feature extraction. Section 4 reports the experimental results on a representative texture database. Section 5 gives the conclusion and future work.

\section{REVIEW OF LOCAL BINARY PATTERN (LBP)}

LBP [7] is a gray-scale texture operator that characterizes the local spatial structure of the image texture. Given a central pixel in the image, a pattern code is computed by comparing its value with those of its neighborhoods:

$$
L B P_{P, R}=\sum_{p=0}^{P-1} s\left(g_{p}-g_{c}\right) 2^{p}, s(x)=\left\{\begin{array}{l}
1, x \geq 0 \\
0, x<0
\end{array}\right.
$$

where $g_{c}$ is the gray value of the central pixel, $g_{p}$ is the value of its neighbors, $P$ is the total number of involved neighbors and $R$ is the radius of the neighborhood. Suppose the coordinate of $g_{c}$ is $(0,0)$, then the coordinates of $g_{p}$ are given 
by $(R \cos (2 \pi p / P), \quad R \sin (2 \pi p / P))$. The gray values of neighbors that are not in the center of grids can be estimated by interpolation.

After the LBP pattern of each pixel is identified, a histogram is built to represent the whole texture image. The $U$ value of an LBP pattern is defined as the number of spatial transitions (bitwise 0/1 changes) in that pattern:

$$
U\left(L B P_{P, R}\right)=\left|s\left(g_{P-1}-g_{c}\right)-s\left(g_{0}-g_{c}\right)\right|+\sum_{p=1}^{P-1}\left|s\left(g_{P}-g_{c}\right)-s\left(g_{p-1}-g_{c}\right)\right|
$$

The uniform LBP patterns refer to the patterns which have limited transition or discontinuities $(U \leq 2)$ in the circular binary presentation [7]. It was verified that only those "uniform" patterns are fundamental patterns of local image texture [7]. To achieve rotation invariance, a locally rotation invariant pattern could be defined as:

$$
L B P_{P, R}^{r i u 2}= \begin{cases}\sum_{p=0}^{P-1} s\left(g_{p}-g_{c}\right) & \text { if } U\left(L B P_{P, R}\right) \leq 2 \\ P+1 & \text { otherwise }\end{cases}
$$

The dissimilarity of sample and model histograms is a test of goodness-of-fit, which could be measured with a nonparametric statistic test. In this study, chi-square distance with the nearest neighborhood classifier is used as it shows good result in texture classification [9].

\section{ADAPTIVE LBP (ALBP) WITH DIRECTIONAL STATISTICAL FEATURES}

\subsection{The directional statistical features}
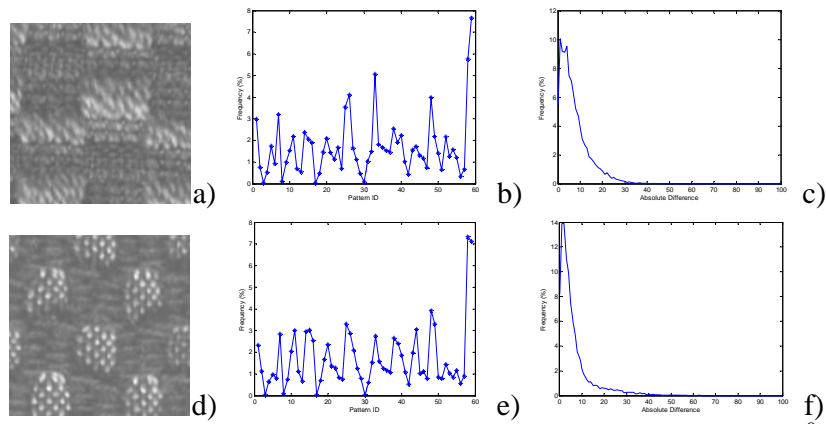

Fig. 1. Similar LBP distributions but different directional $\left(45^{0}\right)$ difference distributions. a)-c) original image, $L B P_{8,1}^{u 2}$ distribution and $\left|g_{c}-g_{1}\right|$ distribution. d)-f) is another example.

The LBP could achieve good texture classification results. However, the local spatial structure could not be fully represented by LBP. Therefore, in [7] the variance (VAR) operator was proposed and used in joint with LBP:

$$
V A R_{P, R}=\frac{1}{P} \sum_{p=0}^{P-1}\left(g_{p}-u\right)^{2}, u=\frac{1}{P} \sum_{p=0}^{P-1} g_{p}
$$

However, $V A R_{P, R}$ has continuous values and it needs to be quantized. This can be done by calculating the feature distribution from all training images and then partitioning the distribution into equally distributed $K$ bins by cut values [7]. The cut values obtained from training images are used for the quantization of test images.

Because VAR is complementary with LBP, their joint distribution could improve the accuracy of texture classification. However, $V A R_{P, R}$ ignores the directional information of local differences because it averages the squares of local differences of the $P$ neighbors from all $P$ directions. In addition, the quantization step may be sensitive to the training samples. When the training samples are less representative, the cut value may fail to partition the feature space evenly.

Given a central pixel $g_{c}$ and its $P$ circularly and evenly spaced neighbors $g_{p}, p=0,1, \ldots, P-1$. The distributions of the differences between $g_{c}$ and $g_{p}$ often vary with $p$, which represents different orientations. Fig. 1 shows an example, the two texture images have similar LBP distributions but different distributions of local difference $\left|g_{c}-g_{p}\right|$. We calculate the mean and standard deviation (std) of $\left|g_{c}-g_{p}\right|$ :

$$
\begin{gathered}
\mu_{p}=\sum_{i=1}^{N} \sum_{j=1}^{M}\left|g_{c}(i, j)-g_{p}(i, j)\right| /(M * N) \\
\sigma_{p}=\sqrt{\sum_{i=1}^{N} \sum_{j=1}^{M}\left(\left|g_{c}(i, j)-g_{p}(i, j)\right|-\mu_{p}\right)^{2} /(M * N)}
\end{gathered}
$$

Suppose the texture image is of size $N \times M$. Where $g_{c}(i, j)$ is the center pixel at position $(i, j)$ and $g_{p}(i, j)$ is neighborhood of $g_{c}(i, j)$ lying along orientation $2 \pi p / P$ with radius $R$. Obviously, $\mu_{p}$ and $\sigma_{p}$ are the first-order and second-order directional statistics of $\left|g_{c}-g_{p}\right|$ along orientation $2 \pi p / P$. We denote by $\vec{\mu}=\left[\mu_{0}, \mu_{1}, \ldots, \mu_{P-1}\right]$ the mean vector and by $\vec{\sigma}=\left[\sigma_{0}, \sigma_{1}, \ldots, \sigma_{P-1}\right]$ the std vector. Fig. 2 shows an example. We can see that with the rotation of the texture image, the mean vector $\vec{\mu}$ and std vector $\vec{\sigma}$ will shift accordingly.

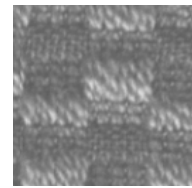

a) $0^{0}$ Sample

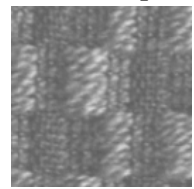

b) $90^{\circ}$ Sample

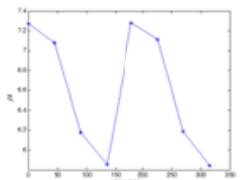

c) $\vec{\mu}$ of a)

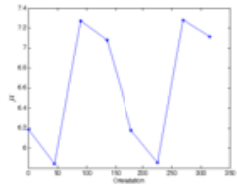

d) $\vec{\mu}$ of b)

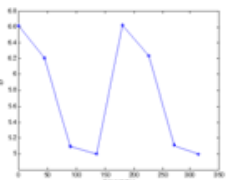

e) $\vec{\sigma}$ of a)

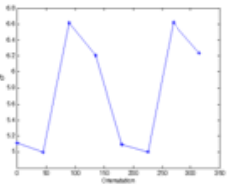

f) $\vec{\sigma}$ of b)
Fig. 2. An example to illustrate that when the image is rotated, the statistical vectors $\vec{\mu}$ and std vector $\vec{\sigma}$ will shift accordingly. Here $P=8$ and $R=1$.

The directional statistical features $\vec{\mu}$ and $\vec{\sigma}$ carry useful information for texture discrimination. Since they are complementary to LBP, we can use them to weight the LBP dissimilarity. As shown in Fig. 2, $\vec{\mu}$ and $\vec{\sigma}$ are variant to orientation changes. There are two possible ways to achieve rotation invariance. I) Given two $\vec{\mu}$ (or two $\vec{\sigma}$ ) from two images, we could shift one of them so that the two vectors have minimal distance. II) Find a dominant orientation for each vector and shift the vector with respect to this orientation. The dominant orientation could be evaluated by the minimal element of the vector. Our tests show that the 
second way has comparable alignment accuracy to the first way but it has much less computational cost.

Denote by $\vec{\mu}_{S}$ and $\vec{\sigma}_{S}$ the directional statistical feature vectors from image $S$, and by $\vec{\mu}_{T}$ and $\vec{\sigma}_{T}$ the vectors from the model $T$. Suppose these vectors have been aligned by either one of the two methods mentioned above. The normalized distances between $\vec{\mu}_{S}$ and $\vec{\mu}_{T}$, and $\vec{\sigma}_{S}$ and $\vec{\sigma}_{T}$ are defined as

$$
d_{u}=\sum_{p=0}^{P-1}\left|\vec{u}_{S}(p)-\vec{u}_{T}(p)\right| /\left(P^{*} \kappa_{\mu}\right), d_{\sigma}=\sum_{p=0}^{P-1}\left|\vec{\sigma}_{S}(p)-\vec{\sigma}_{T}(p)\right| /\left(P^{*} \kappa_{\sigma}\right)
$$

where $\kappa_{u}$ and $\kappa_{\sigma}$ are standard deviation value of $\vec{\mu}$ and $\vec{\sigma}$ from training samples [12].

With $d_{u}$ and $d_{\sigma}$, the weighted LBP dissimilarity is defined as

$$
\begin{aligned}
D_{L B P}^{F}(S, T)= & D_{L B P}(S, T) *\left(1+c_{1}-c_{1} \cdot \exp \left(-d_{u} / c_{2}\right)\right) \\
& *\left(1+c_{1}-c_{1} \cdot \exp \left(-d_{\sigma} / c_{2}\right)\right)
\end{aligned}
$$

where $D_{L B P}(S, T)$ is the LBP histogram dissimilarity, $c_{1}$ and $c_{2}$ are parameters to control the weights and decay speed. For simplicity, identical weights for two features are used here, although better accuracy could be gotten if different weights are used.

\subsection{Adaptive LBP (ALBP)}

To better use $\vec{\mu}$ and $\vec{\sigma}$ to improve the texture classification performance, intuitively we want to minimize the variations of the mean and std of the directional differences. To this end, an adaptive LBP (ALBP) scheme is proposed here to minimize the directional difference along different orientation. Specifically, we introduce a parameter $w_{p}$ so that the overall directional difference $\left|g_{c}-w_{p}{ }^{*} g_{p}\right|$ can be minimized. The objective function is as follows:

$$
w_{p}=\underset{w}{\arg \min }\left\{\sum_{i=1}^{N} \sum_{j=1}^{M}\left|g_{c}(i, j)-w \cdot g_{p}(i, j)\right|^{2}\right\}
$$

Clearly, the least square estimation (LSE) technique can be used for such an optimization and the weight $w_{p}$ can be easily computed as follows:

$$
w_{p}=\vec{g}_{p}^{T} \vec{g}_{c} /\left(\vec{g}_{p}^{T} \vec{g}_{p}\right)
$$

where $\vec{g}_{c}=\left[g_{c}(1,1) ; g_{c}(1,2) ; \ldots ; g_{c}(N, M)\right]$ is a column vector containing all the possible $g_{c}(\mathrm{i}, \mathrm{j})$ pixels and $\vec{g}_{p}=\left[g_{p}(1,1) ; g_{p}(1,2) ; \ldots ; g_{p}(N, M)\right]$ is the corresponding vector for all $g_{p}(\mathrm{i}, \mathrm{j})$ pixels.

Here each weight $w_{p}$ is estimated along one orientation $2 \pi p / P$ for the whole image. Finally, the ALBP is defined as:

$$
A L B P_{P, R}=\sum_{p=0}^{P-1} s\left(g_{p} * w_{p}-g_{c}\right) 2^{p}
$$

Let $\vec{w}=\left[w_{0}, w_{2}, \ldots w_{P-1}\right]$ be the ALBP weight vector. Similar as $\vec{\mu}$ and $\vec{\sigma}$, the ALBP weight vector is also shifted with the rotation of the image. We can align $\vec{w}$ of two images using the same method as that for $\vec{\mu}$ and $\vec{\sigma}$.

\subsection{ALBP with directional statistical features}

With ALBP, the directional statistics in Eqs. (5) and (6) can be computed by:

$$
\begin{gathered}
\mu_{p}=\sum_{i=1}^{N} \sum_{j=1}^{M}\left|g_{c}(i, j)-g_{p}(i, j) * w_{p}\right| /(M * N) \\
\sigma_{p}=\sqrt{\sum_{i=1}^{N} \sum_{j=1}^{M}\left(\left|g_{c}(i, j)-g_{p}(i, j) * w_{p}\right|-\mu_{p}\right)^{2} /(M * N)}
\end{gathered}
$$

Now we have three vectors, the mean $\vec{\mu}$, the std vector $\vec{\sigma}$ and the weight vector $\vec{w}$, which could be used to improve the performance of LBP. Denote by $\left(\vec{\mu}_{S}, \vec{\sigma}_{S}, \vec{w}_{S}\right)$ the vectors from image $S$, and by $\left(\vec{\mu}_{T}, \vec{\sigma}_{T}, \vec{w}_{T}\right)$ the vectors from image $T$. Suppose these vectors have been aligned. Similar to the normalized distances between $\vec{\mu}_{S}$ and $\vec{\mu}_{T}$, and $\vec{\sigma}_{S}$ and $\vec{\sigma}_{T}$ defined in (7), the normalized distance between $\vec{w}_{S}$ and $\vec{w}_{T}$ can be computed as follows:

$$
d_{w}=\sum_{p=0}^{P-1}\left|\vec{w}_{S}(p)-\vec{w}_{T}(p)\right| /\left(P * \kappa_{w}\right)
$$

where $\kappa_{w}$ is standard deviation value of $\vec{w}$ from training samples [12].

Finally, the ALBP dissimilarity weighted by these directional statistical features is defined as:

$$
\begin{aligned}
& D_{A L B P}^{F}(S, T)=D_{A L B P}(S, T) *\left(1+c_{1}-c_{1} \cdot \exp \left(-d_{u} / c_{2}\right)\right. \\
& \quad *\left(1+c_{1}-c_{1} \cdot \exp \left(-d_{\sigma} / c_{2}\right)\right) *\left(1+c_{1}-c_{1} \cdot \exp \left(-d_{w} / c_{2}\right)\right)
\end{aligned}
$$

where $D_{A L B P}(S, T)$ is the ALBP histogram dissimilarity.

Similar as LBP based schemes, the proposed method can be easily extended to multiscale scheme. For example, the simple sum of matching distances under different scales [7] could be used to further improve the accuracy.

\section{EXPERIMENT RESULTS}

This section presents the experimental results on public texture databases to demonstrate the effectiveness of the proposed method. Due to the limit of space, we only show the results on the comprehensive CUReT database [13], which is one of the largest databases for rotation invariant texture classification.

We compare the proposed method with the state-of-theart LBP based schemes in [7] and the state-of-the-art rotation invariant texture classification algorithm MR8 [9]. There are three LBP based feature extraction operators in [7]: $L B P_{P, R}^{r i u 2}, V_{A R}$ and the joint $L B P_{P, R}^{r i u 2} / V A R_{P, R}$. In the experiments, the quantization levels for $V_{A R_{P, R}}$ and $L B P_{P, R}^{r i u 2} / V A R_{P, R}$ were set as 128 and 16 bins as in [7]. In MR8, 10 textons are clustered from each texture class using training samples, and then a histogram based on the $61 * 10$ textons is computed for each model and sample image.

To better illustrate the proposed approach, in the experiments we list all the classification rates by using $A L B P_{P, R}^{r i u 2}$ (i.e. using Eq. (11)), $L B P F_{P, R}^{r i u 2}$ (i.e. using Eq. (8)) and $A L B P F_{P, R}^{r i u 2}$ (i.e. using Eq. (15)). For simplicity, we set $c_{1}=c_{2}=1$ in the experiments, although better accuracy could be achieved if these parameters are tuned appropriately. The code of the proposed method can be downloaded at http://www4.comp.polyu.edu.hk/ cslzhang/code.htm. 
The CURet database contains 61 textures, and each texture has 205 images captured under different viewpoints and illumination conditions. There are 118 images whose viewing angles are less than $60^{\circ}$. Out of the 118 images, 92 images, from which a sufficiently large region could be cropped $(200 * 200)$ across all texture classes, are selected [9]. Except for MR8, all the cropped regions are converted to grey scale and the intensity is normalized to have a mean of 128 and standard deviation of 20 to reduce the variance of illumination [7]. For MR8, the image is normalized to have an average intensity of 0 and a standard deviation of 1 [9].

The training set for each class was built by selecting the first 23 images. Hence, there are 1,403 models and 4,209 testing samples. This setting is close to the situation with a small number of and less comprehensive training sample images. The classification results by different operators of single scale and multiscale are listed in Table 1.

Table 1. Classification rate (\%) using different schemes.

\begin{tabular}{|c|c|c|c|c|}
\hline$P, R$ & 8,1 & 16,3 & 24,5 & $\begin{array}{l}8,1+16, \\
3+24,5\end{array}$ \\
\hline$V A R_{P, R}$ & 44.7 & 39.2 & 37.0 & 47.9 \\
\hline$L B P_{P, R}^{r i u 2} / V A R_{P, R}$ & 70.8 & 70.1 & 65.4 & 73.2 \\
\hline$L B P_{P, R}^{r i u 2}$ & 58.0 & 66.5 & 63.4 & 78.4 \\
\hline$A L B P_{P, R}^{r i u 2}$ & 57.4 & 67.3 & 65.5 & 78.0 \\
\hline$L B P F_{P, R}^{r i u 2}$ & 64.7 & 71.6 & 69.1 & 80.5 \\
\hline$A L B P F_{P, R}^{r i u 2}$ & 66.1 & 72.7 & 71.7 & 80.2 \\
\hline MR8 & \multicolumn{4}{|c|}{77.5} \\
\hline
\end{tabular}

From Table 1 we could make the following findings. First, the proposed directional statistical features are very useful for texture classification. The $L B P F_{P, R}^{r i u 2}$ and $A L B P F_{P, R}^{r i u} 2$ schemes have much better result than $L B P_{P, R}^{r i u 2}$ and $A L B P_{P, R}^{r i u 2}$. Second, $A L B P F_{P, R}^{r i u} 2$ achieves better result than $L B P F_{P, R}^{r i u 2}$. The improvement is more than $1 \%$ in classification rate. This validates the effectiveness of the proposed ALBP scheme. However, multiscale of $A L B P F_{P, R}^{r i u 2}$ fails to get better result than that of $L B P F_{P, R}^{r i u 2}$ by the simple fusion scheme. Better accuracy is expected if an advanced fusion scheme is used. Third, $A L B P F_{P, R}^{r i u 2}$ has comparable result to $L B P_{P, R}^{r i u 2} / V A R_{P, R}$, while the former has a much smaller feature size than the latter. For example, when $P=24$, the feature sizes are 98 (i.e. $26+24 * 3$ ) for $A L B P F_{P, R}^{r i u 2}$ and 416 (i.e. 26*16) for $L B P_{P, R}^{r i u 2} /$ $V A R_{P, R}$. Furthermore, $A L B P F_{P, R}^{r i u 2}$ does not need any quantization step which may fail to represent the whole feature space well when the training samples are not enough and less representative. This is the main reason why $A L B P F_{P, R}^{r i u 2}$ could get $2 \%$ improvement compared with $L B P_{P, R}^{r i u 2}$ $/ V A R_{P, R}$. Finally, by our experiments, using three scales $\{(8,1)+(16,3)+(24,5)\}, A L B P F_{P, R}^{r i u 2}$ could achieve better result than the state-of-the-art algorithm, MR8. Meanwhile, the feature dimension, only 198 (i.e.
$10+8 * 3+18+16 * 3+26+24 * 3$ ), is still much lower that of MR8, which is 610 .

\section{CONCLUSIONS}

LBP has been widely used in texture classification because of its simplicity and efficiency. However, using the local spatial structure feature alone may fail to describe some texture patterns. In this paper, we proposed three kinds of directional statistical features: the mean value and standard deviation of the local directional differences, as well as the adaptive coefficients to minimize the local differences. These statistical features were used to weight the LBP histogram distances for texture classification. Experimental results on the comprehensive CUReT texture database showed that our proposed feature extraction and matching scheme achieves better result than the state-of-the-art rotation invariant texture classification algorithms. In addition, it has a smaller feature size.

\section{ACKNOWLEDGEMENT}

This research is supported by the Hong Kong PolyU Internal Fund (G-YH54), the National Basic Research Program of China (973 Program, No. 2010CB732506) and NSFC (No. 60972110).

\section{REFERENCES}

[1] M. Tuceryan, and A. K. Jain, Texture analysis, Handbook of pattern recognition and computer vision, Chen, C.H., Pau, L.F., and Wang, P.S.P., eds. Chapter 2, pp. 235-276, 1993.

[2] R.M. Haralik, K. Shanmugam, and I. Dinstein, "Texture features for image classification,” IEEE Trans. on Systems, Man, and Cybertics 3(6), pp. 610-621, 1973.

[3] T. Randen, and J.H. Husy, "Filtering for texture classification: a comparative study,” IEEE Trans. on PAMI 21(4), pp. 291-310, 1999.

[4] R.L. Kashyap, and A. Khotanzed, "A model-based method for rotation invariant texture classification," IEEE Trans. on PAMI 8(4), pp. 472-481, 1986.

[5] J. Mao and A.K. Jain, "Texture classification and segmentation using multiresolution simultaneous autoregressive models," Pattern Recognition 25(2), pp. 173-188, 1992.

[6] W.R. Wu, and S.C. Wei, "Rotation and gray-scale transforminvariant texture classification using spiral resampling, subband decomposition, and hidden Markov model," IEEE Trans. on IP 5(10), pp. 1423-1434, 1996.

[7] T. Ojala, M. Pietikäinen, and T. T. Mäenpää, "Multiresolution gray-scale and rotation invariant texture classification with Local Binary Pattern,” IEEE Trans. on PAMI 24(7), pp. 971-987, 2002.

[8] K. Jafari-Khouzani, and H. Soltanian-Zadeh, "Radon transform orientation estimation for rotation invariant texture analysis," IEEE Trans. on PAMI 27(6), pp. 1004-1008, 2005.

[9] M. Varma, and A. Zisserman, "A statistical approach to texture classification from single images," IJCV 62(1-2), pp. 61-81, 2005.

[10] S. Lazebnik, C. Schmid, and J. Ponce, "A sparse texture representation using local affine regions," IEEE Trans. on PAMI 27(8), pp. 1265-1278, 2005.

[11] Y. Xu, H. Ji, and C. Fermuller, "Viewpoint invariant texture description using fractal analysis,” IJCV 38(1), pp. 85-100, 2005.

[12] B.S. Manjunath, and W.Y. Ma, "Texture features for browsing and retrieval of image data," IEEE Trans. on PAMI 18(8), pp. 837842, 1996.

[13] K.J. Dana, B. van Ginneken, S.K. Nayar, J.J. Koenderink, "Reflectance and texture of real world surfaces," ACM Trans. on Graphics 18(1), pp. 1-34, 1999. 